\title{
Automatic Gait Recognition by Symmetry Analysis
}

\author{
James B. Hayfron-Acquah, Mark S. Nixon and John N. Carter \\ University of Southampton, S017 1BJ Southampton, United Kingdom. \\ \begin{tabular}{|l|l|l|l}
\hline joha99r msn jnc eecs.soton.ac.uk \\
\hline
\end{tabular}
}

\begin{abstract}
We describe a new method for automatic gait recognition based on analysing the symmetry of human motion, by using the Generalised Symmetry Operator. This operator, rather than relying on the borders of a shape or on general appearance, locates features by their symmetrical properties. This approach is reinforced by the psychologists' view that gait is a symmetrical pattern of motion and by other works. We applied our new method to two different databases and derived gait signatures for silhouettes and optical flow. The results show that the symmetry properties of individuals' gait appear to be unique and can indeed be used for recognition. We have so far achieved promising recognition rates of over $95 \%$. Performance analysis also suggests that symmetry enjoys practical advantages such as relative immunity to noise and missing frames, and with capability to handle occlusion.
\end{abstract}

\section{Introduction}

Recently, there has emerged a new application domain of computer vision dealing with the analysis of human images. This includes ear and face recognition, body tracking and hand gesture recognition, to mention just a few. Recently, gait recognition has been added to this domain. As a biometric, gait concerns recognising people by the way they walk. One major advantage of gait over other biometrics (e.g. fingerprints) is that it does not require contact. Further gait is difficult to disguise or conceal, in application scenarios like bank robbery. Currently, gait is also the only biometric at a distance. Though it could be argued that physical condition factors such as drunkenness, pregnancy and injuries involving joints can affect an individual's motion, these factors are similar in principle to factors affecting other biometrics. The aim of gait recognition is to recognise people regardless of the clothes worn or the differing background. There have been allied studies of gait, notably among these are medical studies, psychological studies, modelling human motion and tracking people. Amongst these, psychologists suggest gait is a symmetrical pattern of motion[2] and that humans perceive gait as unique.

Although gait recognition is a fairly new research area, there is already a number of approaches. In the spatio-temporal approach, which is probably the earliest, the gait signature was derived from the spatio-temporal patterns of a walking person[7]. The different patterns of the motions of the head and the legs in translation and time were extracted. The patterns were then processed to determine the motion of the bounding contours from which a five-stick model was fitted. The gait signature was then 
derived by normalising the fitted model in terms of velocity, that is by linear interpolation, and encouraging $(85 \%)$ recognition rates were achieved.

In [4], optical flow was used to derive the gait signature by analysing the motion content (shape of motion) of a human walking. Generic object-motion characterisation is also another approach where the gait signature is derived from a parametric eigenspace[5] and the approach was applied to a database of seven subjects with ten image sequences each. The recognition rates were $88 \%$ and $100 \%$ for 8 and 16 eigenvectors, respectively. The approach was extended[3] to use canonical analysis, a model free approach to reduce the dimensionality of the input data whilst optimising class separability. Recently, Shutler et al extended statistical gait recognition via temporal moments [10]. This derived statistics with an intimate relationship to gait, with symmetry properties. In [6], gait signatures were derived from the frequency components of the variations in the inclination of the human thigh. As pendula modelled the periodic motion of the thigh during walking, this again suggests that symmetry analysis is suited to gait recognition.

\section{Symmetry and its extraction}

Symmetry is a fundamental (geometric) property suggesting it to be an important principle of perception[9]. An object is said be to symmetric if its shape is invariant to the application of symmetry operations. Boolean symmetry operations can only assess symmetry when the shape of the object is known in advance, rendering them inefficient. The discrete symmetry operator can estimate symmetricity without the knowledge of the object's shape, unlike feature extraction operators that find a shape by relying on its border. The symmetry transform assigns a symmetry measure to each point in the image and is determined with respect to a given point-symmetry group. It has also been shown that the performance of the symmetry transform is not affected by existence of several objects in the scene[9].

To extract the symmetry of a walking human subject, feature templates are extracted from gait sequences to give template sequences. The symmetry operator uses an edge map of images in the sequences to assign symmetry magnitude and orientation to image points, accumulated at the midpoint of each pair of points.

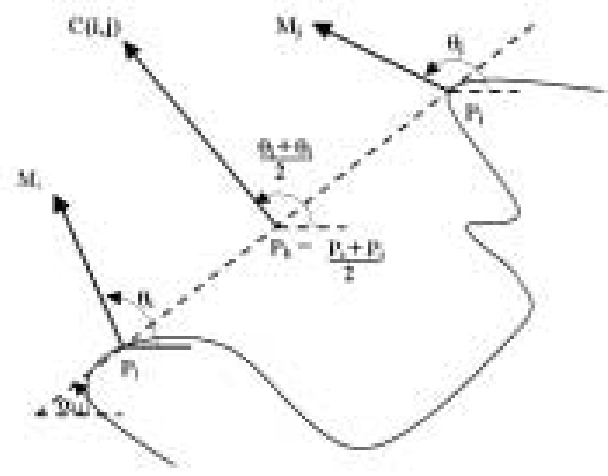

Figure 1. The symmetry contribution of edge points $P_{i}$ and $P_{j}$ 
The symmetry relation or contribution, $C(i, j)$ between the two points $P_{i}$ and $P_{j}$ is:

$$
C(i, j)=D_{i, j} P h_{i, j} I_{i} I_{j}
$$

The symmetry distance weighting function, $D$, is defined as the minimum effort required to turn a given shape into its symmetric shape. It reflects the distance between two different points $P_{i}$ and $P_{j}$, and is calculated as:

$$
D_{i, j}=1 / \sqrt{2 \pi \sigma} \exp \left(-\left(\frac{\left\|P_{i}-P_{j}\right\|}{2 \sigma}-\mu\right), \forall i \neq j\right.
$$

where $\sigma$ controls the scope of the function. Each value of $\sigma$ implies a different scale thus making it suited to multi-resolution schemes. A large value of $\sigma$ implies largescale symmetry that gives distant points similar weighting to close points. Alternatively, a small value of $\sigma$ implies local operation and local symmetry. Recently a focus, $\mu$, was therefore introduced into the distance weighting function to control the focusing capability of the function, hence further improving the scaling possibilities of the symmetry distance function. The addition of the focus into the distance weighting function moves the attention of the symmetry operator from points close together to a selected distance.

The logarithm intensity function, $I_{i}$, of the edge magnitude $M$ at point $(x, y)$ is $I_{i}=\log \left(1+M_{i}\right)$. Using the logarithm of magnitude reduces the differences between high gradients or symmetries resulting from weak edges, making the correlation measure less sensitive to very strong edges. The phase weighting function between two points $P_{i}$ and $P_{j}$ is:

$$
P h_{i, j}=\left(1-\cos \left(\theta_{i}+\theta_{j}-2 \alpha_{i, j}\right)\right)\left(1-\cos \left(\theta_{i}-\theta_{j}\right)\right), \quad \forall i \neq j, \alpha(i, j)=\operatorname{atan}\left(\frac{y_{i}-y_{j}}{x_{i}-x_{j}}\right)
$$

is the angle between the line joining the two points and the horizon. The symmetry contribution value obtained is then plotted at the midpoint of the two points. The symmetry transform as discussed here detects reflectional symmetry. It is invariant under 2D rotation and translation transformations and under change in scale [9], and as such has potential advantage in automatic gait recognition.

\section{Symmetry and gait}

\subsection{Deriving the gait signature}

To derive the gait signature for a subject an image sequence is used. The following gives an overview of the steps involved. First, the image background is subtracted from the original image, Fig. 2a to obtain the silhouette, Fig. 2b. The Sobel operator is then applied to the image in Fig. 2b to derive its edge-map, Fig. 2c. Where the gait signature is derived from optical flow information, the optical flow image is extracted from two successive silhouettes. The edge-map is thresholded so as to set all points beneath a chosen threshold to zero, to reduce noise or remove edges with weak strength, which may be due to the background removal. The symmetry operator is then applied to give the symmetry map, Fig. $2 \mathrm{~d}$. For each image sequence, the gait signature, $G S$, is obtained by averaging all the symmetry maps. 


\subsection{Gait recognition}

The Fourier transform was then applied to each of the gait signatures and the transform was low-pass filtered to reduce sensitivity to high-frequency components.

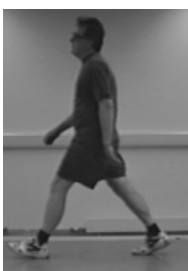

(a) original

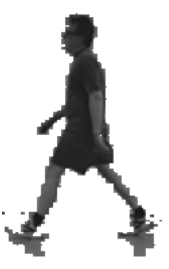

(b) silhouette

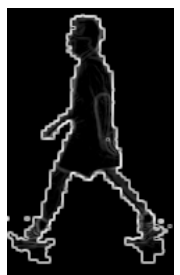

(c) after Sobel

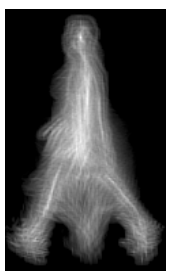

(d) symmetry map

Figure 2 Images from the SOTON data

Different cut-off frequencies were used to determine the appropriate number of Fourier components. For purposes of classification or recognition, the similarity differences between the Fourier descriptions of the gait signatures are then calculated using Euclidean distance. The magnitude spectra only were used here because they gave a better result than by using phase.

\subsection{Recognition by symmetry}

The new method was applied to two different databases of spatial templates. The SOTON database has four subjects with four image sequences each and that of UCSD six subjects with seven image sequences of each. For both SOTON and UCSD databases, we derived gait signatures for silhouette and optical flow information. These provide alternative versions of the input data for our technique to process. The values for $\sigma$ and $\mu$ used were 27 and 90, respectively, unless otherwise stated. The $k$ Nearest Neighbour rule was then applied for classification, using $k=1$ and $k=3$, as summarised in Table 1. The correct classification rates were $100 \%$ for both $k=1$ and $k=3$ for the SOTON database. For the UCSD database, the recognition rates for silhouette information were 97.6 and $92.9 \%$ for $k=1$ and $k=3$. A CCR of $92.9 \%$ was obtained for the optical flow information, for both $k=1$ and $k=3$.

\begin{tabular}{|c|c|c|c|cc|}
\hline \multirow{2}{*}{ Database } & \multirow{2}{*}{ \# Subjects } & \multirow{2}{*}{ \# Sequences } & \multirow{2}{*}{ Data Type } & \multicolumn{2}{|c|}{ CCR $(\%)$} \\
\cline { 4 - 6 } & & & $k=1$ & $k=3$ \\
\hline \multirow{2}{*}{ SOTON } & \multirow{2}{*}{4} & \multirow{2}{*}{16} & Silhouette & 100 & 100 \\
\hline \multirow{2}{*}{ UCSD } & \multirow{2}{*}{6} & \multirow{2}{*}{42} & Optical flow & 100 & 100 \\
& & Silhouette & 97.6 & 92.9 \\
& & Optical flow & 92.9 & 92.9 \\
\hline
\end{tabular}

Table 1: Initial results obtained from two disparate databases.

For the low pass filter, all possible values of radius were used to investigate the number of components that can be used (covering 0.1 to $100 \%$ of the Fourier data). Though the results of Table 1 were achieved for all radii greater than 3 (using the SOTON database), selecting fewer Fourier components might affect the recognition rates on a larger database of subjects, and this needs to be investigated in future. 


\subsection{Performance analysis of symmetry operator}

Performance was evaluated with respect to missing spatial data, missing frames and noise using the SOTON database. Out of the 16 image sequences in the database, one (from subject 4) was used as the test subject with the remainder for training.

Missing frames: The evaluation, aimed to simulate time lapse, was done omitting a consecutive number of frames. For a range of percentages of omitted frames, Fig. $3 \mathrm{a}$, the results showed no effect on the recognition rates for $k=1$ or $k=3$. This is due to the averaging associated with the symmetry operator. Fig. 3a shows the general trend of deviation of the best match of each subject to the test subject.

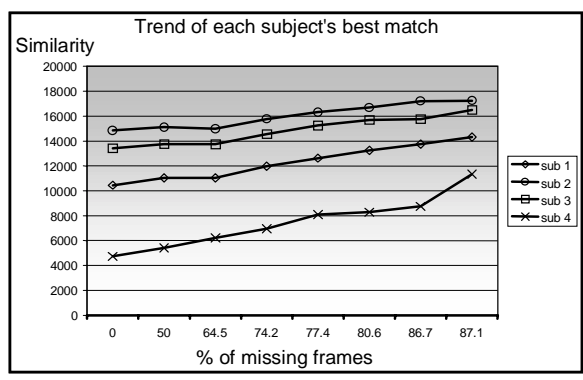

(a) effect of missing image frames

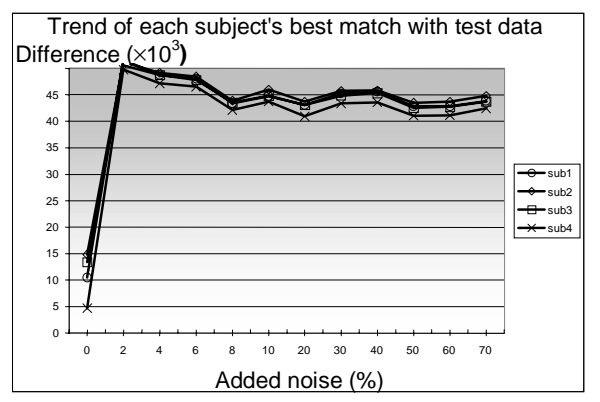

(b) effect of adding noise Figure 3 Performance Analysis: Omitted Frames and Addition of Noise

Adding/Omitting spatial data: The evaluation was done by masking with a rectangular bar of different widths: 5, 10 and 15 pixels in each image frame of the test subject and at the same position. The area masked was on average $13.2 \%, 26.3 \%$ and $39.5 \%$ of the image silhouettes, respectively. The bar either had the same colour as the image silhouette or as the background colour, as shown in Fig. 4, simulating omission and addition of spatial data, respectively. In both cases, recognition rates of $100 \%$ were obtained for bar size of 5 pixels for both $k=1$ and $k=3$. For a bar width of 10 pixels, Fig. 4c failed but Fig. 4a gave the correct recognition for $k=3$ but not for $k=1$. For bar sizes of 15 and above, the test subject could not be recognised.

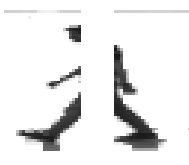

(a) 10 pels

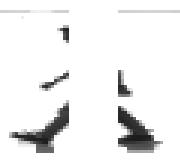

(b) 15 pels

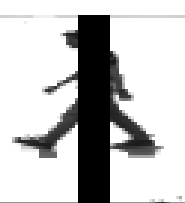

(c) 10 pels

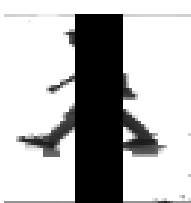

(c) 15 pels

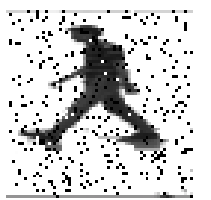

(e) $10 \%$

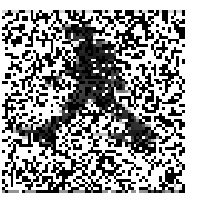

(f) $50 \%$

Figure 4. Occluded and Noisy Data

Noise: To investigate the effects of noise, we added synthetic noise to each image frame of a test subject and compared the resulting signature with those of the other subjects in the database. Fig. 4 shows samples of the noise levels used. The evaluation was carried out under two conditions. First by using the same values of $\sigma$ and $\mu$ (eqn. 2 ) as earlier. For a noise level of 5\%, the recognition rates for both $k=1$ and $k=3$ were $100 \%$. For $10 \%$ added noise, the test subject could still be recognised correctly 
for $k=1$ but not for $k=3$. With added noise levels of $20 \%$ and above, the test subject could not be recognised for $k=1$ or $k=3$. With the second condition, the values of $\sigma$ and $\mu$ were made relatively small. The recognition rates $(100 \%)$ were not affected for both $k=1$ and $k=3$ for added noise levels even exceeding 60\%. Fig. 3b shows how the best match of each subject deviated from the test subject as more noise was added.

\section{Conclusions}

The aim of this paper is to support the psychology view that the symmetry of human motion can be used for recognition. We have therefore presented, as a starting point, a new approach to automatic gait recognition. It has been shown that human gait appears to have distinct symmetrical properties that can be extracted for recognition. The symmetry operator, essentially, forms an accumulator of points, which are measures of the symmetry between image points to give a symmetry map. By using the symmetry operator, the Discrete Fourier Transform and a basic nearest-neighbour approach, the results have produced a recognition rate of $100 \%$ for both $k=1$ and $k=3$ on a small database of four subjects. Comparable recognition rates have been achieved using the same databases as in other works. The symmetry operator has been shown to handle missing spatial data, missing image frames, and to some extent noise. Thus, it will prove very useful when applied to poorly extracted sequences, partially occluded and missing frames in image sequences for gait recognition.

\section{References}

1. D. Cunado, M. S. Nixon and J. N. Carter, "Gait extraction and description by evidence gathering”, Proc. 2nd Int. Conf AVBPA99, Washington, 1999, pp 43-48

2. J. T. Cutting, D. R. Proffitt and L. T. Kozlowski, "A biomechanical invariant for gait perception”, J. Exp. Psych.: Human Perception and Performance, 1978, pp 357-372

3. P. S. Huang, C. J. Harris and M. S. Nixon, "Human gait recognition in canonical space using spatio-temporal templates", IEE Proc. VISP, April 1999, pp 93-100

4. J. Little and J. Boyd, "Recognizing People by Their Gait: The Shape of Motion", Videre, 1(2), 1-32, 1998

5. H. Murase and R. Sakai, "Moving object recognition in eigenspace representation: gait analysis and lip reading", Patt. Recog. Lett., 1996, pp 155-162

6. M. S. Nixon, J. N. Carter, P. S. Huang and S. V. Stevenage, "Automatic Gait Recognition", In: BIOMETRICS Personal identification in Networked Society, Chapter 11, pp 231-250. Kluwer Academic Publishers, 1999

7. S. A. Niyogi and E. H. Adelson, "Analysing and recognising walking figures in xyt", In: Proc. CVPR, 1994, pp 469-474

8. C. J. Parsons and M. S. Nixon, "Introducing focus in the generalised symmetry operator", IEEE Sig. Proc. Lett., 3, 1999, pp 49-51

9. D. Reisfeld, H. Wolfson, and Y. Yeshurun, "Context-free attentional operators: The generalised symmetry transform". IJVC, 1995, pp 119-130

10. J. D. Shutler, M. S. Nixon and C. J. Harris, "Statistical gait recognition via temporal moments". 4th IEEE Southwest Symp. on Image Analysis and Int., 2000, pp 291-295 